\title{
UN ENTOMOLOGO IN INDIA: L'ORIENTALISMO CONSAPEVOLE DI GUIDO GOZZANO
}

\author{
GIUSEPPE GAZZOLA \\ (Stony Brook University, New York)
}

\begin{abstract}
This article analyzes Guido Gozzano's collection of Indian letters, Verso la cuna del mondo (Towards the Cradle of Mankind, 1917). After taking a position in the recent debate concerning the authorial standing of the book, the essay demonstrates the exceptionality of Gozzano's case within the genre of Orientalism writing. Thanks to his ironic mode, the poet is able to develop an early awareness of Orientalist tópoi, and succeeds in manipulating them according to his own expressive needs.
\end{abstract}

Sarebbe forse azzardato asserire che Verso la cuna del mondo sia il testo più conosciuto di Guido Gozzano: ma, di questi tempi, è quello più stampato. Certo l'argomento è attraente; le prose di Verso la cuna del mondo (sottotitolo: Lettere dall'India 1912-1913) vennero scritte al ritorno da un viaggio in India compiuto da Gozzano nel 1911 e durato una cinquantina di giorni, che nella finzione letteraria diventò un'esplorazione di quasi tre mesi ${ }^{1}$. I racconti, pubblicati inizialmente su "La Stampa" di Torino e su alcuni periodici a tiratura regionale e nazionale tra il 1914 e il 1916, furono poi raccolti in un'edizione

Aletta Grisay ha elaborato una cronologia di entrambi i viaggi, quello reale e quello descritto, nel fondamentale "L'India di Guido Gozzano e quella di Pierre Loti" apparso su La rassegna della letteratura italiana, settembre-dicembre 1967:427-437. Altrettanto utili sono le ricostruzioni di Alida D'Aquino Creazzo, in 'La letteratura dell'esotismo e Guido Gozzano", (Le ragioni critiche, ottobre-dicembre 1978:333-355) e nelle note dell'edizione di Verso la cuna del mondo da lei curata (Firenze: Olschki, 1984). A quest'ultimo libro si rimanda anche per l'elenco delle pubblicazioni dei testi su quotidiani e periodici. 
postuma dagli editori Treves nel 1917, con la prefazione di Giuseppe Antonio Borgese ${ }^{2}$.

Fino agli anni Settanta del Novecento il libro è apparso solo insieme ad altre opere; ma dagli anni Ottanta in avanti le edizioni si sono susseguite senza apparente soluzione di continuità, in italiano, in inglese e in tedesco. Le edizioni sono, purtroppo, molto diverse tra loro, e talvolta rimaneggiate. I vari curatori sono in disaccordo su una questione, letteralmente, fondamentale: se l'editio princeps del libro sia da considerarsi autoriale o meno. Alcuni propendono per il sì, come Alida D'Aquino Creazzo e Flaminio di Biagi: ${ }^{3}$ Gozzano prima di morire avrebbe avuto il tempo di selezionare e ordinare gli articoli, rivederli sommariamente, apportare delle piccole varianti, decidere il titolo del libro, insomma di conferire l'imprimatur. Altri propendono per il no, rifacendosi tra gli altri all'autorità di Giorgio De Rienzo, che ha curato l'edizione apparsa per gli Oscar Mondadori nel 1983: e il libro sarebbe allora un'iniziativa del fratello minore Renato, o dell'editore, o del prefatore, o di tutti costoro messi insieme.

Evidentemente tale tradizione bicipite ha dato origine a edizioni molto diverse tra loro: alcuni curatori si sono sentiti autorizzati a modificare il titolo del libro e l'ordine e il numero dei capitoli secondo criteri tematici, geografici, cronologici o narrativi, e inoltre a non rispettare le variazioni apportate in volume agli articoli apparsi su rivista.

Bisogna dire che il disaccordo tra $\mathrm{i}$ critici a riguardo dell'ordinamento del corpus gozzaniano non si limita al testo di Verso la cuna del mondo, e già Calcaterra e De Marchi, curando la prima edizione integrale delle Opere, ebbero a lamentare:

Le principali raccolte poetiche, curate dall'autore ( $L$ a via del rifugio e I colloqui), sono state più volte manomesse, sconvolte, alterate nella struttura e nel testo; le opere postume sono state trasformate ad arbitrio, spesso

2 I riferimenti al testo e le citazioni proverranno da tale edizione, d'ora in avanti VCM.

3 Dell'edizione curata da Alida D'Aquino Creazzo si è detto; l'edizione a cura di Flaminio di Biagi, con postfazione di Giorgio Bárberi Squarotti, è apparsa per i tipi di La Finestra, Lavis (Tn), 2005. 
condotte ad altri sensi nella dizione, stilisticamente ritoccate in più luoghi ed emendate; le rime sparse e le prose disperse sono state in complesso mal riprodotte, rimanipolate e più di una volta interpretate a vanvera. Dalla folla stessa dei lettori si sono levati insistenti richiami a maggior fedeltà e a miglior cura ${ }^{4}$.

Il fatto che nell'agosto del 1943 gli archivi della casa editrice Garzanti, che contenevano i documenti ancora inediti riguardanti gli ultimi anni di vita di Gozzano, siano andati distrutti in un incendio non aiuta la curiosità dei lettori: i quali, nel caso del libro indiano, in mancanza di prove autografe hanno potuto cimentarsi in una squisita discussione filologica.

Chi scrive è stato convinto dell'autorità dell'editio princeps non solo dall'acribia di Alida D'Aquino Creazzo, ma anche dal ben noto intuito del primo prefatore, che cominciò la sua introduzione-trenodia con queste parole: "È bene che il lettore, chiuso questo libro del nostro caro morto Guido Gozzano, indugi un poco prima di giungere ad una conclusione sul suo significato e sul suo valore. / Udrà allora molti suoni fievoli e sordi comporsi in una triste armonia seduttrice; vedrà molte macchie di colore, che parevano buttate a caso, connettersi pei margini e formar quadro" .

Le Lettere formano infatti un quadro completo, e talvolta si richiamano l'un l'altra secondo un ordine cronologico intenzionale, come si avrà modo di osservare più avanti. Mi sentirei inoltre di affermare che Borgese, nel caso fosse intervenuto sul testo, non avrebbe avuto remore nel pretendere la dovuta parte di merito per aver trasformato in libro ciò che libro non era.

In ogni caso - che si creda o meno all'autorità dell'edizione Treves- rimane incontrovertibile che, a partire dagli anni Settanta, da quando cioè un famoso libro di Edward Said ha posto una nuova definizione di orientalismo al centro del dibattito critico

4 Da "Le opere e il poeta", in Guido Gozzano: Opere, a cura di Carlo Calcaterra e Alberto De Marchi. Nuova edizione riveduta e aumentata. Milano: Garzanti, 1956:vii.

5 Così inizia la prefazione di G.A. Borgese a VCM:v (riprodotta anche nell'edizione citata a cura di Flaminio di Biagi). 
internazionale $^{6}$, Verso la cuna del mondo ha fruito di un rinnovato interesse da parte del pubblico dei lettori. Il fatto può destare meraviglia quando si pensi che libri sullo stesso argomento, scritti da studiosi molto più preparati di Gozzano e pubblicati solo qualche anno prima, giacciono nelle biblioteche: non ristampati, poco letti, e considerati ancor meno ${ }^{7}$.

Perché il libro indiano di Gozzano desta oggi più interesse del volume di Paolo Mantegazza, antropologo esimio, o dei ponderosi tomi di Angelo de Gubernatis, professore di sanscrito, che pure durante il loro momento di gloria ebbero un dignitoso numero di ristampe ${ }^{8}$ ? Perché, diversamente dai propri predecessori, Gozzano rappresenta per noi un tipo di orientalista che Said non aveva previsto: l'orientalista consapevole.

Mettere in relazione il caso Gozzano con la più vasta interpretazione del fenomeno orientalista può portare ad interessanti considerazioni su Gozzano stesso: in primis, l'utilizzazione d'autore dell'orientalismo come mezzo di definizione del sè. L'ironia che questa lettura ci porta a evidenziare, inoltre, è la stessa resa celebre dalla Via del rifugio e da I colloqui; ritrovarla in Verso la cuna del mondo apre la strada ad una considerazione importante: per il Gozzano esotico, esattamente come per il poeta o per l'autore di novelle, scrivere significa operare su altra letteratura, fare metaletteratura.

Che Gozzano sia, fin dai primi tentativi letterari, suscettibile al fascino dell'esotico è fatto conosciuto, analizzato e riscontrabile

6 Edward Said: Orientalism. New York: Vintage Books, 1978.

7 Prezioso nella sua eccezionalità è il volume di letture critiche di Ernesto Citro, Percorsi indiani: Mantegazza, De Gubernatis, Lomonaco, Gozzano. Roma: Bulzoni, 2006.

8 I volumi in questione sono: Paolo Mantegazza, India. Milano: Treves, quarta edizione 1888. Angelo de Gubernatis: Peregrinazioni indiane. In tre volumi. Firenze: Niccolai, 1887. Grazie al progetto googlebooks, entrambi i libri sono adesso disponibili in rete. A proposito del successo dei libri al momento della loro pubblicazione, si legga quanto scrive Mantegazza nella prefazione alla quarta edizione del suo India: "[...] il mio libro al di qua e al di la delle Alpi si è fatto un posto al sole e tra tanti fratelli più saputi di lui si è messo a sedere, senza alcuna voglia di muoversi di lì, anzi mormorando superbamente: hic manebimus optime. E invece quei poveri Profili e paesaggi della Sardegna, scritti con tanto amore per la Sardegna e per i Sardi, scritti con tanti anni di meno sulle spalle, son rimasti alla prima edizione. Perché? Habent sua fata libelli" (dall'edizione citata:VI). 
nell'intero libro di Roberto Carnero, Guido Gozzano esotico ${ }^{9}$. Carnero giustamente segnala "l'antico bramino dei Pattarsy" che si incontra appena imboccata l'eponima Via del rifugio, come secondo termine di una similitudine:
Sognare! Il sogno allenta
la mente che prosegue:
s'adagia nelle tregue
l'anima sonnolenta,
siccome quell'antico
brahamino dei Pattarsy
che per racconsolarsi
si fissa l'umbilico ${ }^{10}$.

Nel poemetto immediatamente successivo si legge la narrazione dell'ottuagenario protagonista de L'Analfabeta, che racconta al poeta bambino di Odessa e Gerusalemme, e del Sultano di Costantinopoli; Gozzano usa termini che attirano la nostra attenzione e ci mostrano come la città che interessa il giovane Guido sia "fittizia", una città da stampa o da cartolina.

Allora che su questa soglia stessa mi narravi di guerre e d'altri popoli, dicevi del Mar Nero e Sebastopoli, dei Turchi, di Lamarmora, d'Odessa.

E nel mio sogno s'accendean le vampe sopra le mura. Entrava la milizia

nella città: una città fittizia

quali si vedon nelle vecchie stampe,

\footnotetext{
Roberto Carnero, Guido Gozzano esotico. Anzio: De Rubeis, 1996.

Guido Gozzano, Opere cit.:10.
} 
le vecchie stampe incorniciate in nero:

[...] i panorami di Gerusalemme,

il Gran Sultano, carico di gemme [...]:

artificiose, belle più del vero;

le vecchie stampe, care ai nostri nonni $[\ldots]$ il minareto e tre colonne infrante, il mare, la galea, il mercatante [...] città vedute nei miei primi sonni. ${ }^{11}$

Le vecchie stampe (l'espressione è tanto importante da essere ripetuta tre volte), per poter essere più belle del vero, non possono essere vere; appaiono, infatti, in un viaggio onirico. Già dalle prime prove l'orientalismo di Gozzano suona "un poco falso": ed il giudizio viene confermato da un testo composto solo un paio di anni prima del viaggio in India, il poemetto Paolo e Virginia stampato su rivista nel dicembre 1910 e poi incluso nei Colloqui.

Come si capisce fin dal titolo, il poemetto è la riscrittura in tono crepuscolare del più famoso romanzo di Bernardin de Saint-Pierre, Paul et Virginie, ${ }^{12}$ in cui i protagonisti - due bimbi francesi cresciuti in perfetta libertà nella colonia di Mauritius - si innamorano, ma vengono crudelmente separati quando Virginie viene mandata in patria dai genitori per studiare. Nonostante l'abbandono, Paul la aspetta sull'isola, paziente: e quasi impazzisce di gioia quando il vecchio tutore lo informa che Virginie sta ritornando da lui. Ma il veliero su cui Virginie sta viaggiando fa naufragio sulla scogliera sotto gli occhi di Paul che, disperato, si lascia morire. Gozzano nella propria riduzione non degna di eccessiva gravitas i sottesi sociali del romanzo (l'esistenza o meno dell'idea russoviana del perfetto stato di natura, che renderebbe inutile la divisione sociale europea in classi; o la questione morale causata nei due ragazzi dalla pratica coloniale della schiavitù), e si concentra invece sullo svolgimento romantico e tragico dell'intreccio. Nella seconda strofe, in cui Paolo si rivolge

11 Ibidem:18.

12

Charles Augustin Sainte-Beuve Bernardin de Saint-Pierre, in Paul et Virginie - La Chaumière indienne. Paris: Curmer, 1858 (prima edizione 1787). 
direttemente a Virginia (che è già morta, secondo la prolessi tra fabula ed intreccio ripresa dall'originale), si legge:
Virginia ti rammenti
di quella sempiterna primavera?
Rammenti i campi d'indaco e di the, e le Missioni e il Padre e il Viceré, quel Tropico rammenti, di maniera, un poco falso, come piace a me? [...]
Ti rammenti il colore
del Settecento esotico, l'odore
di pace, filtro di non so che frutto
e di non so che fiore,
il filtro che dismemora di tutto? $[\ldots]^{13}$

Alla voce di Paul si è qui sovrapposta quella di Guido: il Paul di Bernardin de Saint-Pierre, eroe sentimentale ma affatto privo di sussulti d'autocoscienza, non avrebbe potuto esprimersi in questo modo. Invece l'esotismo caro a Gozzano è quello "un poco falso", riconosciuto nel suo sentimentalismo melico, ingenuo e fantastico. Riscrivendo un libro altrui, Gozzano è consapevole di riprodurre sulla carta un incantevole mondo di maniera, incantevole proprio perché di maniera, come i boschi nelle fiabe. E dalla messa in scena prende le distanze, come autore, attraverso due artifici: una personalissima manipolazione delle fonti, e l'uso dell'ironia.

Se queste sono le premesse, non ci si stupirà di quanto avviene in Verso la cuna del mondo, il testo in cui ci troviamo di fronte ad uno scrittore pienamente orientalista. Eppure, un orientalista atipico: perché, diversamente dai viaggiatori che lo hanno preceduto, egli dimostra di essere conscio del fatto che la sua scrittura sull'India sia soltanto una rappresentazione, solo un'opera di letteratura. La coscienza di essere parte di un fenomeno culturale e di compiere un'operazione letteraria crea una grande distanza fra l'autore e la realtà del viaggio: Gozzano, mentre scrive un libro di argomento

13 Da Paolo e Virginia - I figli dell'infortunio (in I colloqui, dove precede il celeberrimo poemetto La signorina Felicita ovvero La Felicità). Guido Gozzano, Opere cit.:100. 
orientalista, può permettersi di guardare la propria opera da una prospettiva critica.

Non si può proporre, come si è fatto per autori della letteratura italiana più illustri di Gozzano, una completa divisione di caratteri tra il pellegrino e l'autore: se non altro perché le necessità di pubblicazione degli articoli prima e del libro poi richiedevano una completa sovrapposizione dei personaggi. Ma almeno una cosa va detta: mentre il Gozzano viaggiatore usa le proprie fonti come una sorta di Baedeker spirituale per orientarsi nell'India del contrasti e dei paradossi, il Gozzano scrittore usa a volte queste stesse fonti per sorridere dei luoghi comuni che l'orientalismo ha diffuso intorno all'India.

Vediamo un paio di esempi ricalcati sui tanti che Aletta Grisay propone come risultato della propria ricerca: Pierre Loti scrive nel capitolo "Chez le Maharajah du Travancore" del suo L'Inde (sans les Anglais):

Ce pays, par endroits, me réservait donc la surprise de ressembler au mien, de me rendre, en plein hiver, le charme de nos fins d'été [...] Et, sans tout à fait oublier au fond de moi-même que je suis dans l'Inde, en un lieu perdu, je me livre, avec une mélancolie douce, à des illusions de terre natale. Les campagnes de l'Aunis ou de la Saintonge, les tranquilles demeures de l'île d'Oleron à la saison lumineuse et dorée des vendanges, me sont rappelées nostalgiquement par ces horizons plats, ces petits murs blancs, ces jasmins, cette herbe jaunie et ces couleurs d'automne. Mille détails toutefois traversent mon rêve pour le dérouler. Un passant nu, qui frôle sans bruit les graminées du chemin, me montre un fin visage de couleur sombre. Un colibri, près des moineaux, vient se poser avec un éclat de pierre précieuse ${ }^{14}$.

\section{E Gozzano riscrive:}

14 Pierre Loti: L'Inde (sans les Anglais). Paris: Calman-Lévi, 1903:42. 
Esco tra le aiuole ben pettinate, dove le rose bengali s'alternano con ortaggi europei, tanto che in questo mattino di gennaio ho l'illusione di passeggiare in un giardino canavesano, nelle nostre più belle giornate estive; ma una frotta di pappagalli verdi, una farfalla troppo ampia e troppo abbagliante, inconciliabile col nostro cielo, mi ricorda il tropico, mi dà l'incubo quasi dell'estate sempiterna. Giunge di lontano un suono discorde e assiduo di tam-tam, di gonghi, di pifferi, che sovrasta il suono delle campane cattoliche, un'orchestra selvaggia che mi parla di misteri paurosi e d'idolatria ${ }^{15}$.

Come ha osservato Aletta Grisay, Gozzano rielabora un'idea già presente nello scritto usato come fonte: l'illusione di un paesaggio europeo viene messa in forse da alcuni dettagli e poi annientata da un ultimo particolare talmente estraneo da rendere impossibile ogni paragone. Ma è tutto gozzaniano il contrasto ironico fra i fiori esotici, le "rose bengali", ed un particolare europeo assai meno poetico, "gli ortaggi"; un'ulteriore variazione sul tema del contrasto tra aulico e prosastico. Più oltre, la descrizione ambientale tratta da un capitolo di Loti ("Dans l'Inde des grandes palmes") viene resa secondo stilemi familiari a qualsiasi lettore di poesia crepuscolare. Scrive Loti:

En plus des officiers et fonctionnaires de la colonie, on trouve ici quelques familles créoles, arrivées à l'époque éroïque et devenues tout à fait pondichériennes après quatre ou cinq générations. Vieilles dames aux gentilles manières un peu surannées. Vieux salons d'un charme un peu mélancolique, avec leurs fauteuils du XVIII siècle, avec leurs pendules Louis XVI ou Empire, aventureusement venues jadis par le cap de BonneEspérance, alors que l'on ne prévoyait pas encore le transit égyptien, et ayant compté les heures de tant d'existences languides, ayant précisé la minute de tant d'agonies en exil [...]. C'est enfantin sans doute, mais les

15 VCM: Da Ceylon a Madura:88. 
pendules d'autrefois, rencontrées aux colonies, arrêtent toujours longuement ma pensée $[\ldots]^{16}$

\section{E Gozzano:}

A tavola, solo. La saletta mi dà qualche illusione d'Europa, illusione che accresce, non mitiga la mia nostalgia. È singolare il contrasto fra la lindezza tropicale, le pareti bianche di calce, traforate a mezzo, fino al soffitto, e la pesantezza presuntuosa e vetusta dello scarso arredo che ricorda le sale d'aspetto di certi dottori o di certi curati; quattro sedie in giunco, un divano esalante da troppe ferite l'anima di stoppa, una mensola Impero con sopra un pendolo Robert di qualche pregio, uno scaffale con una Bibbia enorme, alle pareti un'oleografia moderna dei reali d'Inghilterra e due incisioni antiche: Amsterdam del secolo VII ${ }^{17}$;

Come non ricordare le "buone cose di pessimo gusto" del salotto di Nonna Speranza: le stampe, i cofani, l'orologio a cucù, le miniature e i "daggherottìpi" che facevano rinascere Guido nel 1850? Ciò che a Aletta Grisay sembra costruito su uno spunto di Loti è in realtà un salotto gozzaniano trasportato ai tropici. E si noti che, come nella Amica di Nonna Speranza, il distacco ironico all'inizio della descrizione dell'arredo (assente nel Loti) sfocia poi in toni più decisamente fiabeschi e sognanti.

Ma l'ironia di Verso la cuna del mondo non è unicamente costruita a spese delle fonti, anzi sono numerosi i passaggi in cui questa nasce da riflessioni autonome. Nella lettera collocata in apertura di volume, "Le grotte della Trimurti", Gozzano racconta la sua visita a Garapuri, o Isola d'Elefanta, e le riflessioni che i bassorilievi gli suggeriscono. Così scrive del complesso dei templi:

16 Pierre Loti: L'Inde (sans les Anglais) cit. :215.

17 VCM: Un Natale a Ceylon:75. 
Sebbene mutilato dai millenni, dalle infiltrazioni e dalle frane, dal fanatismo mussulmano e portoghese, presenta ancora una sintesi completa e imponente dell'olimpo brahamino; olimpo complicatissimo, difficile a chiarire per chi non ha speciali attitudini a collegare le famiglie numerose. Domina nella grotta principale un altorilievo di forse quindici metri, raffigurante un corpo formidabile a tre teste, la Trimurti famosa: Siva che crea, Wisnu che conserva, Rudra che distrugge. Ma questa trinità s'incarna all'infinito, si trasforma nei bassorilievi dei porticati semibui in mille altre figure $\mathrm{d}[\mathrm{a}] \mathrm{l}$ simbolismo pazzesco. Ed ecco Siva che cavalca un toro e si fa maschio e femmina ad un tempo, col simbolo maschile linga, e femminile joni, circondato da infinite figure: elefanti, tigri, serpenti, da saggi, rhisi, da apsare, uri dell'olimpo brahamino, da Indra, da Brahma, adagiato sul loto e portato da quattro cigni, Visnu sorridente, altovolante sull'avvoltoio dalla testa umana. [...]Un altro bassorilievo rappresenta un giardino: il paradisiaco monte Kaillasa, pieno di saggi e di donne in letizia, poiché dall'unione di Siva con Parvati è nato Ganesa, il Dio della Sapienza, mostro dalla testa di elefante, dal corpo umano, piccolino, tondeggiante, panciuto. ¿̀ ancora Siva in un bassorilievo che ritrae le più desolanti e borghesi rappresaglie di famiglia che possono affliggere un nume. Siva ha sposato una seconda moglie: Durga, figlia di Daksha, figlio di Bhraham e genitore di sessanta figliole; Daksha dà un convito rituale, aduna tutti gli Dei e dimentica sciaguratamente il genero Siva e consorte. Questa interviene al rito e, non attesa, male accolta, si getta sulle fiamme dell'ara. Compare Siva, al quale nel furore si moltiplicano le braccia, e taglia la testa al suocero, alle cinquantanove figlie, ai convitati con lo spaventoso congegno delle molte braccia roteanti; intorno è un turbinare di teste mozze $[\ldots]^{18}$.

18 VCM: Le grotte della Trimurti:13-14. 
La descrizione dei templi dell'isola di Elefanta va considerata nella sua interezza, perché si capisca come Gozzano affronti la civiltà hindu (nel suo primo compito di giornalista: tradurre in parole le conoscenze e le emozioni trasmessegli da ciò che vede) mettendo le mani avanti: ciò che sta per spiegare è "complicatissimo, difficile a chiarire" a chi non sia in possesso di speciali virtù onomastiche. Ma improvvisamente di tali virtù difetta lo stesso autore, che confonde una divinità con un'altra e sostituisce a Brahma il nome di Rudra ${ }^{19}$, confonde un'aquila con un avvoltoio ${ }^{20}$, e confonde la mente dei lettori non avendo capito e sapendo di non voler capire. Tanto è vero che a un certo punto sbotta: "è certo il mio cervello profano d'occidentale che non comprende l'occulto senso della pietra scolpita" ${ }^{21}$.

Si prenda ad esempio l'episodio dell'insulto a Shiva da parte di Daksha, che è raccontato nel volume Santi Parva del Mahabharata ${ }^{22}$. Qui la figlia di Daksha, che si chiama Uma e non Durga (Durga è una differente emanazione di Shakti, e la sua storia non ha nulla a che fare con nessuna delle storie qui ricordate) è una emanazione di Shakti tanto quanto Parvati. Gozzano non capisce, o capisce e non vuole spiegare, l'idea fondamentale della mitologia hindu: il concetto di unità alla base della molteplicità. L'unione di Shiva e Shakti rappresenta l'unione delle forze vitali maschile e femminile: Uma e Parvati sono entrambe versioni di Shakti, che è la forza femminile elementare. Rendere questo concetto in termini di prime o seconde nozze di Shiva riporta ad una concezione europea della famiglia completamente inadeguata a descrivere la situazione del mito, e allo

19 Tutte le rappresentazioni della Trimurti comprendono Shiva, Visnu, e Brahma. Rudra, correttamente indicato come dio della distruzione, è una emanazione di Shiva.

20 Garuda, divinità prediletta da Visnu come cavalcatura in tempo di battaglia e rappresentato sia in forma di aquila con testa umana che in forma di uomo con testa aquilina, non può essere confuso con un avvoltoio, la cui razza è alleata dei demoni.

21 VCM: Le grotte della Trimurti:14.

22 Cfr. The Mahabharata, tradotto in inglese da Kisari Mohan Ganguli, in www.sacredtexts.com/hin/ml2/index.htm. Il passo in questione è nel libro 12, sezione CCLXXXIV:317. 
stesso tempo confonde la fantasticata (dagli europei) poligamia musulmana con la rigida monogamia hindu.

Difficile sarebbe cogliere in fallo Loti, Mantegazza o de Gubernatis nelle descrizioni dei templi da loro visitati; ma a Gozzano non interessa tale tipo di accuratezza, facilmente ottenibile acquistando un libro in inglese $\mathrm{o}$ in francese all'agenzia Cook di Bombay, o ascoltando con un po' di attenzione le spiegazioni della guida. Gli interessa invece raccontare la storia a modo proprio, con la leggerezza che gli è consona, come aveva già fatto con il testo di Bernardin de Saint-Pierre: e pazienza se alcuni dettagli vanno perduti o si confondono. Nella sua ottica, lo sterminio attuato da Shiva a spese del pantheon brahamino per vendicare la moglie suicidatasi per futilissimi motivi non è nulla di più che una "desolante e borghese rappresaglia di famiglia"; l'ironia, da cui trapela l'incomprensione e l'estraneità dell'Occidentale nei confronti di una realtà diversa e non riconducibile ai suoi consueti parametri di giudizio, è l'arma retorica con cui lo scrittore prende le distanze da ciò che gli sta intorno, dal luogo comune dell'esotico così spesso trattato dai narratori di professione.

D'altronde, Gozzano non si considera uno di questi narratori: nel capitolo "Un Natale a Ceylon" il lettore trova la spiegazione della presenza in India di un così strano viaggiatore: egli è un letteratoentomologo, venuto in quelle regioni a caccia di farfalle esotiche; non un erudito, nè un giornalista, tantomeno uno storico o un esploratore: bensì un collezionista di insetti innocui, una figura che non deve essere presa troppo sul serio: ed è solo il caso a mettergli davanti cobra, tigri e pantere, palazzi principeschi e necropoli immense, cioè a dire tutto l'armamentario con cui si può costruire un immaginario orientale degno di questo nome. Solo il caso: e, forse, le aspettative dei lettori delle riviste su cui le Lettere dall'India furono pubblicate, che non avrebbero potuto immaginare una descrizione dell'India senza i tesori di Golconda, l'Impero del Gran Mogol o il Taj Mahal.

Le descrizioni arrivano, puntuali, nei rispettivi capitoli. Però la prospettiva dell'entomologo è sovente straniata, insolita, fuori tono: proprio il capitolo dedicato a Golconda, città morta nel regno d'Haiderabat, ci offre un significativo esempio. Gozzano comincia la 
descrizione secondo le regole, sfruttando il fascino evocativo del nome e la potenza imaginifica del paesaggio:

Golconda! Al di là d'un gran fiume asciutto s'innalza il fantasma della città morta, con le sue mura ciclopiche, livide come il macigno circostante [...] Il genio guerresco ha trovata qui la collaborazione della natura, nè si può distinguere dove l'opera di questa finisca e cominci lo sforzo dell'uomo. L'uomo ha utilizzato macigni di cinquanta metri, rivestendoli di ammattonato, gettando dall'uno all'altro volte e terrapieni, unendoli con grate grosse come un braccio umano, armate di uncini difensori. Veramente Golconda doveva nascondere tesori favolosi se i Sultani pensarono a cingerla d'una difesa tanto formidabile ${ }^{23}$.

Ma subito dopo l'attenzione del viaggiatore smarrisce la descrizione storica, ed introduce nel discorso un elemento estraneo:

Golconda fioriva ancora nella metà del Settecento, quando era di moda in Europa il racconto d'avventure, le roman merveilleux, quando vi giunse profuga Madama Angot per tentare con la sua bellezza occidentale le stanche voglie dei sultani decrepiti.

Profanazione dei ricordi! La grazia tracotante della pescivendola parigina mi perseguita mentre il professore mi commenta le vicende epiche e i monumenti famosi ${ }^{24}$.

Madama Angot! Ecco un nome ancor più evocativo ed imaginifico! Il personaggio della pescivendola avventuriera creato da AntoineFrançois Ėve sull'onda della rivoluzione francese era proprio il tipo di viaggiatrice adatto ad affascinare Gozzano. Popolana bella e coraggiosa, non aveva paura, ad esempio, di viaggiare in areostato:

23 VCM: I tesori di Golconda:141-142.

24 VCM: I tesori di Golconda:145. 
En ballon elle monte,

La voilà dans les airs;

Et plus tard elle affronte

Les mers et les déserts.

Au Malabar, captive,

La croyant veuve, hélas!

On veut la brûler vive,

C'est la mode là-bas! $!^{25}$

Madame Angot, piena di risorse, non si era persa d'animo neppure quando gli Hindu del Malabar, credendola vedova, tentarono di arderla viva. Il suo personaggio aveva conosciuto una seconda giovinezza da quando Charles Lecocq, compagno di studi e rivale di Bizet nel mondo dell'opéra-comique parigina, aveva portato sulle scene l'operetta La fille de Madame Angot. Il testo era così famoso che a Torino nel 1911, in occasione della rappresentazione dello spettacolo, ne era stata pubblicata un'edizione in italiano.

Non che il collezionista di farfalle avesse bisogno della traduzione italiana, beninteso: tra le righe ci fa capire di avere una certa familiarità con il corpus della poissarde, e soprattutto con il già citato coro della quarta scena del primo atto, che si conclude:

Enfin, toute sa vie,

Elle a voyagé, mais

C'est surtout en Turquie,

Qu'elle eut un vrai succès.

Malgré ses cinq cent femmes,

Le sultan, certain soir,

Brûlant de mille flammes...

Lui jeta le muchoir! $!^{26}$

A Golconda questi versi perseguitano il viaggiatore a tal punto che egli non riesce a liberarsene "mentre il professore gli commenta i

25 La fille de Madame Angot; musica di Charles Lecocq, parole di Louis-François Clairville, Paul Siraudin e Victor Konig. New York: Metropolitan, 1879:9.

26 La fille de Madame Angot cit.:10. 
monumenti famosi". Ironia del caso o ironia d'autore? Sono parole che illustrano bene il concetto di esotismo un poco falso, di maniera, verso cui Gozzano aveva mostrato entusiasmo. Mentre alla voce della guida è riservato il compito di narrare la storia della città perduta $\mathrm{a}$ tutto vantaggio dell'erudizione dei lettori, il poeta rimugina i versi dell'operetta, e finalmente li parafrasa in chiusura di capitolo:

Ma ancora qui la mia malinconia è rallegrata dalla figura della pescivendola avventuriera. È veramente esistita quella che la leggenda chiama Madama Angot e fa pellegrinare ad Algeri, a Costantinopoli, a Golconda?

Illustre pescivendola - era Madama Angot.

Nel regno di Golconda - un giorno capitò;

il gran Sultan vedutala - se ne invaghì così che a cinquecento mogli - lei sola preferì $[\ldots]$

[...]

Madama Angot [...] È veramente esistita? In quest'ora, tra queste mura la sua gaia figura è più viva che mai, serve a consolare d'ogni troppo leopardiana tristezza. Dinanzi alle ruine troppo riverite è consigliabile l'irriverenza ${ }^{27}$.

La costruzione retorica è esemplare per definire la consapevolezza che Gozzano dimostra verso il concetto di Orientalismo: si trovano sulla stessa pagina elementi orientali di maniera e la loro parafrasi, il canone e la variazione. Mentre la guida parla di tesori e di saccheggi, l'attenzione dell'entomologo è tutta intenta a rielaborare i versi di un'operetta ottocentesca, rilocalizzando la posizione geografica della protagonista per soddisfare il proprio bisogno.

La situazione non cambia nella Lettera seguente, "L'impero dei Gran Mogol": a stimolare l'irriverenza dell'autore arrivano, dopo il fantasma di Madama Angot, due 'cattive signorine' in carne ed ossa. Sono, costoro, due Francesi conosciute a Bombay, e ritrovate per caso durante il viaggio di trasferimento verso Agra: "Giovani, una giovanissima, parigine entrambe -parigine di Marsiglia o di Bordeaux-

27 VCM: I tesori di Golconda:147. 
e nate all'arte, votate all'arte, hanno pellegrinato come 'duettiste' tutti i caffè chantants della Tunisia e dell'Egitto. A Port-Said un impresario le ha scritturate per le colonie dell'Africa Orientale fino a Zanzibar. Da Zanzibar sono fuggite con due ufficiali inglesi riparando a Bombay" ${ }^{28}$. Più che cantanti di varietà sono veri e propri personaggi letterari, avventuriere d'antan, in relazione evidente con l'illustre pescivendola ${ }^{29}$. Sono personaggi letterari non tanto perché sono, a loro volta, in un libro, ma per diritto ereditario; dall'antenata hanno preso lo spirito, l'arguzia, il bon mot:

-Le Grand Mogol, Madame, qui avait un penchant pour les jolies parisiennes.

-Peut-on le voir, ce monsieur-là?

-È morto da trecento anni.

-Hélàs! Nous arrivons toujours trop tard $[\ldots]^{30}$

Guido trova la risposta alla domanda con cui aveva concluso il capitolo precedente (Madama Angot è veramente esistita, ecco a provarlo la sua discendenza), e soprattutto trova delle guide per le rovine di Agra più consone alla propria sensibilità:

Dubito che gli archeologi soffrano di vertigini poetiche: dubito della sensibilità di coloro che sanno. In questa solitudine s'incontrano sovente figure biondicce ed occhialute di studiosi russi, tedeschi, inglesi che osservano con fiero cipiglio, come sacerdoti indignati, la nostra gaiezza profanatrice. [...] Sente quest'armonia l'inglese eruditissimo - e scortese - che lavora in una baracca prossima e dirige tre scribi indigeni che disegnano e calcolano per non so che restauri governativi? Ho più fiducia nell'entusiasmo e nel buon

\footnotetext{
28 VCM: L'impero del Gran Mogol:155.

"Madama Angot, che ho sognato a Golconda, rivive dunque ancora nelle pronipoti senza paura!” VCM: L’impero del Gran Mogol:155.

30 VCM: L'impero del Gran Mogol:156.
} 
gusto di queste due meretrici di Francia. La più loquace delle due ha delle immagini adorabili.

-J'ai toujours rêvé ce tableau là quand j'étais fillette, sur un coussin de ma tante Veronique! Et voilà qu'il y a vraiment une chose comme ça ${ }^{31}$.

L'inglese eruditissimo, ma senza vertigini poetiche, viene lasciato in disparte in favore delle due signorine: il Gozzano scrittore, attraverso i suoi personaggi (mi ripeto: talmente di maniera da essere l'incarnazione di un'eroina d'opéra-comique) prende sempre più le distanze dalla rappresentazione orientalista dell'Oriente.

D'altronde, come pensa Edoardo Sanguineti nel suo Guido Gozzano. Indagini e letture, l'esotico già agli inizi del Novecento può essere riconosciuto come una merce culturale di consumo. ${ }^{32}$ Gozzano è ben cosciente delle modalità espressive dell'orientalismo, che nella sua analisi risalgono al Settecento teatrale di Antoine-François Ève, e sa come adoperarle. Per questo il racconto dell'esperienza in India si mostra criticamente consapevole della tradizione che lo precede: per questo ci troviamo di fronte ad un'autore che manipola la maniera esotica, invece di esserne manipolato.

L'intervallo di tempo che si frappone tra il viaggio e il momento in cui le Lettere vennero effettivamente scritte, la falsificazione dei dati (e delle date) che riguardano l'itinerario, e la insistita riscrittura delle fonti fanno pensare che lo scrittore non abbia realizzato una narrazione della propria esperienza, quanto una metanarrazione ponderata dell' accaduto ed elaborata in prima persona.

Il nostro viaggiatore, allora, è solo sulla carta (solo sulla carta del proprio libro) un ingenuo osservatore; è ingenuo solo come personaggio. Come autore, anche quando decida di lasciarsi coinvolgere nel gioco dell'esotismo, mantiene la posizione distaccata e critica di chi sa di fare proprio un atteggiamento letterario, un comportamento culturale.

Sono queste le coordinate entro cui si delinea il "caso" del Gozzano delle Lettere dall'India: la sua esperienza di viaggio ha

31 VCM: L'impero del Gran Mogol:161-162.

Edoardo Sanguineti, Guido Gozzano. Indagini e letture. Einaudi: Torino, 1966:136 e segg. 
bisogno di essere riscritta, inverata e falsificata ad un tempo attraverso la consapevolezza del medium letterario per essere trasformata in un'altra esperienza: estetica, poetica, testuale. "Per Gozzano malato di letteratura non c'è altro di vero che la letteratura": cioè a dire che se la letteratura per il Gozzano viaggiatore "precede

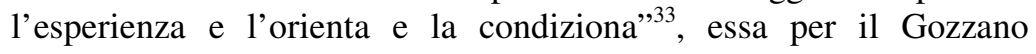
scrittore si fa veicolo di riflessione sulla realtà: per questo Gozzano si sente libero di descrivere l'India non come è, ma come lui l'ha immaginata, sia sui libri di chi lo ha preceduto che durante l'esperienza diretta.

Mentre si diverte a spese dei luoghi comuni sull'esotico e scrive, dopo aver visto a teatro La figlia di Madama Angot, delle sue pronipoti, egli rende il proprio lavoro un veicolo di riflessione sulla letteratura stessa e dimostra che la questione orientalista, per lui, non è altro che letteratura, con mezzo secolo di anticipo rispetto ai ragionamenti di Said. Non sorprenda, dunque, se il pubblico dei lettori trova il libro così adatto ai nostri tempi.

33 Luigi Surdich: Guido Gozzano. L'avventura esotica. Modena: Mucchi, 1993:17. 